\title{
Carbapenem resistant Enterobacteriaceae - the basics for every medical specialty
}

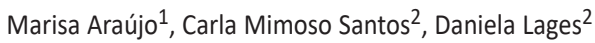 \\ ${ }^{1}$ Hospital Lusíadas Lisboa \\ ${ }^{2}$ Centro Hospitalar Universitário Lisboa Norte, EPE - Hospital de Santa Maria - Serviço de Doenças Infeciosas
}

\section{ABSTRACT}

Enterobacteriaceae are ubiquitous gram-negative bacilli, part of the intestinal flora. The number of carbapenem resistance in this class has been increasing, and K. pneumoniae carbapenemase (KPC) is the most common carbapenemase. Although less frequent, New Delhi metallo-beta-lactamase (NDM-1) is an even more concerning carbapenemase, because of the extremely limited treatment options. The most important risk factor for colonization or infection with such pathogens is the use of broad spectrum cephalosporins and/or carbapenems. Strategies to treat carbapenemases-producing organisms depend not only on susceptibility tests, minimum inhibitory concentration (MIC) and severity of the infection, but also on the availability, costs and policies of antibiotics in each hospital/country. Hospitalized patients, infected or colonized with carbapenemase-producing bacteria, should be placed on contact precautions.

Keywords: antimicrobial therapy, carbapenem resistant Enterobacteriaceae, carbapenemases, KPC-producing Klebsiella pneumonia, multidrug resistance

\section{INTRODUCTION}

Antimicrobial resistance is reaching concerning numbers worldwide. If the rate of increase maintains constant, by 205010 million people will die every year due to antimicrobial resistance ${ }^{1}$.

\section{Carbapenems}

Carbapenem are very broad-spectrum beta-lactam antibiotics. There are four drugs in this class: ertapenem, meropenem, imipenem and doripenem (the latter not marketed in Portugal). Ertapenem distinguishes itself from other carbapenems as it doesn't act on Pseudomonas spp, and is therefore appropriate to treat Gram-negative infections, where others antibiotic classes are not useful, because it doesn't create selective pressure on Pseudomonas spp.

\section{Enterobacteriaceae}

Enterobacteriaceae are ubiquitous gram-negative bacilli, part of the intestinal flora of most animals, including humans. They cause a variety of diseases in humans, including $25-33 \%$ of all bacteremia, over $70 \%$ of urinary tract infections (UTIs), and many intestinal/intra-abdominal infections ${ }^{2}$.

Enterobacteriaceae include several bacterial species (namely Escherichia coli, Klebsiella spp, Proteus spp, Citrobacter spp,
Enterobacter cloacae, Morganella morganii, Serratia marcensces), many with intrinsic antimicrobial resistance. Examples are the SPICE bacteria (Serratia, Providencia, "indole-positive" Proteus species, Citrobacter, Enterobacter species), that have inducible chromosomal $\mathrm{AmpC}$ beta-lactamase genes that may be derepressed during therapy, conferring in vivo beta-lactam resistance (except cefepime and carbapenems) despite apparent sensitivity in vitro ${ }^{3}$.

In addition to intrinsic resistances, Enterobacteriaceae have the ability to acquire new genetic material by mobile genetic elements. This leads to the emergence of new strains with a distinct genetic repertoire, and the acquisition of new antimicrobial resistance mechanisms ${ }^{2}$.

\section{Carbapenemases}

The number of extrinsic resistances has been emerging and increasing. Carbapenemases are carbapenem-hydrolysing beta-lactamases that confer resistance to a broad spectrum of beta-lactam substrates, including carbapenems, and they can arise from previously carbapenemase-negative strains.

Carbapenemases are rapidly spreading worldwide and fall into three main groups: KPC enzymes, belonging to Ambler class $A$; MBLs, belonging to molecular class $B$ and including NDM, VIM, and IMP enzymes, among many others; and OXA enzymes, belonging to class D (in Enterobacteriaceae, OXA-48 is the most prevalent one) (Fig. 1) (,5. $^{4}$. 


\section{Figure 1}

Ambler's molecular classification system ${ }^{5}$

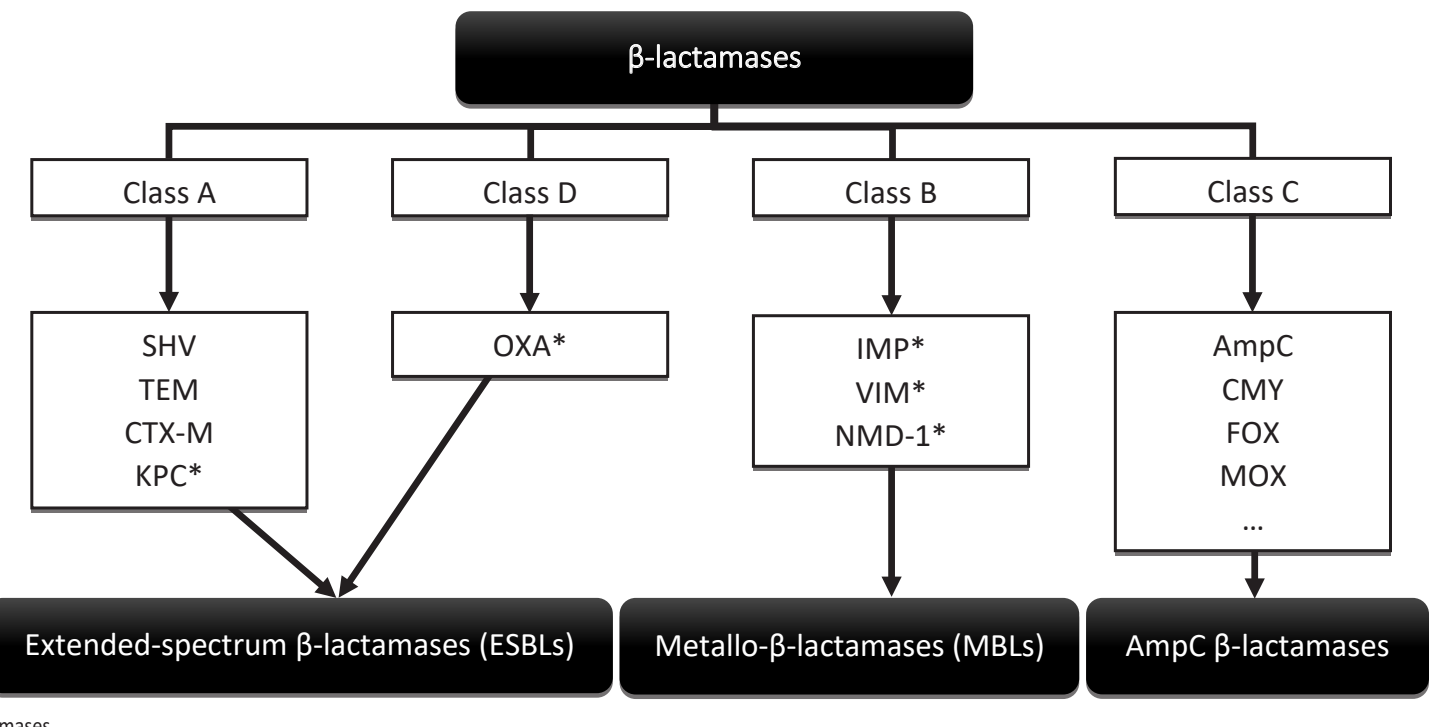

*Also carbapenemases.

\section{EPIDEMIOLOGY}

K. pneumoniae carbapenemase (KPC) is the most common carbapenemase. Following the first description of KPC from a clinical isolate of $K$. pneumoniae in the late 1990s in North Carolina - USA ${ }^{6,7}$, KPC-production has been identified in isolates almost all over the world. The CDC reported that the proportion of Enterobacteriaceae that were carbapenem resistant increased from 1 to 4 percent between 2001 and 2011; the proportion of carbapenem-resistant Klebsiella increased from 2 to 10 percent $^{8}$. The proportion of carbapenem-resistant Klebsiella pneumoniae isolates in Portugal in 2017 was $8.6 \%{ }^{9}$.

The New Delhi metallo-beta-lactamase (NDM-1) was first described in December 2009 in a K. pneumoniae isolate from a Swedish patient who had been hospitalized in India ${ }^{10}$. Subsequent reports have included patients who have travelled and undergone procedures (so called "medical tourism") in India and Pakistan ${ }^{11}$, as well as cases reported in Asia, Europe, North America, the Caribbean, and Australia ${ }^{11-16 .}$

\section{RISK FACTORS}

The most important risk factor for colonization or infection with these pathogens is the use of broad spectrum cephalosporins and/ or carbapenems ${ }^{17-20}$.

Other risk factors include advanced age, poor functional status, severe illness (trauma, malignancy), diabetes, healthcare-associated infection, extended hospitalization (less than three months ago), ICU hospitalization, mechanical ventilation, recurrent/obstructive UTI, genitourinary or biliary instrumentation, immunodepression, organ transplantation, surgical intervention or wound care ${ }^{21-29}$. Clinicians should be also aware of the possibility of NDM-1-producing Enterobacteriaceae in patients who have received medical care in India and Pakistan $^{12}$.

\section{TREATMENT}

Antibiotic options to treat infection due to carbapenemases-producing organisms are limited (Table I).

\section{$\underline{\text { Table I }}$}

Typical resistances and sensibilities of carbapenemase-producing Enterobacteriaceae $e^{4,30}$

\begin{tabular}{l|l}
\multicolumn{1}{c|}{ Usually resistant } & \multicolumn{1}{c}{ Usually sensitive } \\
\hline Carbapenems & Colistin \\
Piperacillin/Tazobactam $\rightarrow 100 \%$ & Tigecycline \\
Ciprofloxacin $\rightarrow 98 \%$ & Aminoglycosides \\
Tobramycin $\rightarrow 94 \%$ & Fosfomycin \\
Cefepime $\rightarrow 60 \%$ & Ceftazidime/Avibactam \\
& Meropenem/Vaborbactam \\
\hline
\end{tabular}

\section{Why not treat with a single antibiotic to which the carbapenemases-producing organisms are susceptible?}

Data suggest that combination therapy may be beneficial for high-risk patients as it has a protective effect on mortality, and also suggest that monotherapy may be enough for lower-risk patients ${ }^{31-34}$. Thereby, low-risk infection (defined as having an INCREMENT mortality score less than eight points) can be treated with monotherapy according to susceptibility 4 . It is worth mentioning that ceftazidime/avibactam or meropenem/vaborbactam was not used in these studies. 


\section{Antibiotics}

\section{Carbapenems}

Carbapenemases-producing organisms are usually resistant to carbapenems, but can we treat Enterobacteriaceae KPC infections with these antibiotics when minimum inhibitory concentration (MIC) suggests susceptibility? The answer is yes: we can indeed treat Enterobacteriaceae KPC infections with carbapenems, depending of MIC. Low risk infection ${ }^{4}$ can be treated with carbapenems in monotherapy if MIC is $4 \mathrm{mg} / \mathrm{L}$ or less. High risk infections (defined as having septic shock or, for bloodstream infections, an INCREMENT mortality score of eight or more points) should be treated with combined therapy between a carbapenem and another active antibiotic if the MIC is 8 $\mathrm{mg} / \mathrm{L}$ or less; for superior MIC, avoid carbapenems.

Double carbapenem therapy can be prescribed when no other active antibiotic is available, using ertapenem as a "suicide substrate" 35 (in these cases, consider $2 \mathrm{~g}$ daily of ertapenem) ${ }^{4}$.

The dose of meropenem should be the double of the usual dose: $2 \mathrm{~g}$ every 8 hours by extended infusion.

Carbapenems have been associated with central nervous system (CNS) adverse effects, including confusional states and lower seizure thresholds, so it should be used with caution with CNS disorders (e.g., brain lesions and history of seizures) and the dose should be adjusted in renal impairment to avoid drug accumulation.

\section{Colistin}

Colistin (polymyxin E) is part of the polymyxins antibiotics; polymyxin B is not available in Portugal. Polymyxins have bactericidal activity and renal excretion.

The recommended doses are listed in Table II.

\section{Table II}

Intravenous colistin (colistimethate sodium) dosing guideline for the treatment of multidrug resistant Gram-negative infections. eGFR = estimated glomerular filtration rate ${ }^{36}$

\begin{tabular}{|c|c|c|}
\hline Dose & Patient category & Dosing suggestion \\
\hline Loading & Critically ill or severe sepsis & $9-12 \mathrm{MU}$ \\
\hline \multirow[t]{6}{*}{ Maintenance } & eGFR $>60 \mathrm{~mL} / \mathrm{min}$ & 4.5 MU 12-hourly \\
\hline & eGFR $30-60 \mathrm{~mL} / \mathrm{min}$ & 3 MU 12-hourly \\
\hline & eGFR $10-30 \mathrm{~mL} / \mathrm{min}$ & $2 \mathrm{MU} 12$-hourly \\
\hline & $\mathrm{eGFR}<10 \mathrm{~mL} / \mathrm{min}$ & $1 \mathrm{MU}$ 12-hourly \\
\hline & Intermittent haemodialysis & $\begin{array}{l}1 \mathrm{MU} \text { 12-hourly plus } \\
\text { supplemental dose of } 1 \mathrm{MU} \\
\text { after each episode of dialysis }\end{array}$ \\
\hline & Continuous renal replacement & 4.5 MU 12-hourly \\
\hline
\end{tabular}

Because polymyxins are cationic polypeptides ${ }^{37}$, they displace $\mathrm{Mg}^{2+}$ and $\mathrm{Ca}^{2+}$ out of cells, and ionogram vigilance should be regularly assessed during treatment.

Some adverse effects include nephrotoxicity and neurotoxicity (confusion, ataxia, vertigo, facial paralysis).

\section{Tigecycline}

Tigecycline have a bacteriostatic activity and should be avoided in monotherapy. Combined therapy is mostly recommended in intrabdominal infections when the MIC is less than $1 \mathrm{mg} / \mathrm{L}$, and the recommended dose is $100 \mathrm{mg}$ for loading dose and then $50 \mathrm{mg}$ every 12 hours. When used in other kind of infections (pneumonia, UTI, bloodstream infections), the dose of tigecycline should be doubled: $200 \mathrm{mg}$ loading dose and then $100 \mathrm{mg}$ every 12 hours ${ }^{4}$.

Adverse effects include hepatotoxicity, with lower fibrinogen blood levels, and therefore this should be monitored.

\section{Aminoglycosides}

With bactericidal activity, they only should be used if MIC is less than $8 \mathrm{mg} / \mathrm{L}$.

It can be used in monotherapy only in UTI. Their use should be avoided when treating abscesses as it doesn't penetrate the pus, because of its low $\mathrm{pH}$.

The usual dose for gentamicin is $5-7 \mathrm{mg} / \mathrm{kg} /$ day and for amikacin is $15-20 \mathrm{mg} / \mathrm{kg} /$ day. For hospital-acquired pneumonia or shock without other options, higher doses might be considered (gentamicin 10-15 $\mathrm{mg} / \mathrm{kg}$, amikacin $25-30 \mathrm{mg} / \mathrm{kg}$ ), but the risk of toxicity is high. For both, therapeutic drug monitoring should be performed regularly ${ }^{4}$.

Nephrotoxicity and ototoxicity are the major adverse effects.

\section{Fosfomycin}

Fosfomycin has a broad spectrum and a bactericidal activity. It is excreted unaltered by urine, and has a quick tissue distribution (it doesn't bind to proteins), so it is a good choice almost everywhere: soft tissue, kidney, lung, bone, central nervous system and cardiac valves.

The recommended dose is between $4 \mathrm{~g}$ every 6 hours and $8 \mathrm{~g}$ every 8 hours, intravenous. For intermittent hemodialysis, it could be administrated 2 to $4 \mathrm{~g}$ after each episode of dialysis. Of note, intravenous fosfomycin is not available in many countries.

It can induce hypokalemia, but this can be overcome through perfusions longer than two hours. It should be noted that it has a high sodium concentration (every gram of fosfomycin has $0.33 \mathrm{mg}$ of sodium).

\section{Ceftazidime/Avibactam}

This also has a broad spectrum and a bactericidal activity. There are only a few studies into its use.

The use of ceftazidime-avibactam alone or in combination should be evaluated. On the one hand, KPC-3-producing Klebsiella (the most common in Portugal) are vulnerable to mutations in the enzyme causing resistance ${ }^{38}$; on the other hand, two studies observed restoration of meropenem susceptibility among ceftazidime/avibactam resistant Klebsiella pneumoniae ${ }^{39}$. Therefore, it must be considered whether ceftazidime/avibactam should be used with a carbapenem to treat infections with KPC, especially in the KPC-3 producers ${ }^{38}$.

The recommended dose is $2.5 \mathrm{~g}$ every 8 hours. 


\section{Figure 2}

What to do after carbapenem resistant Enterobacteriaceae detection

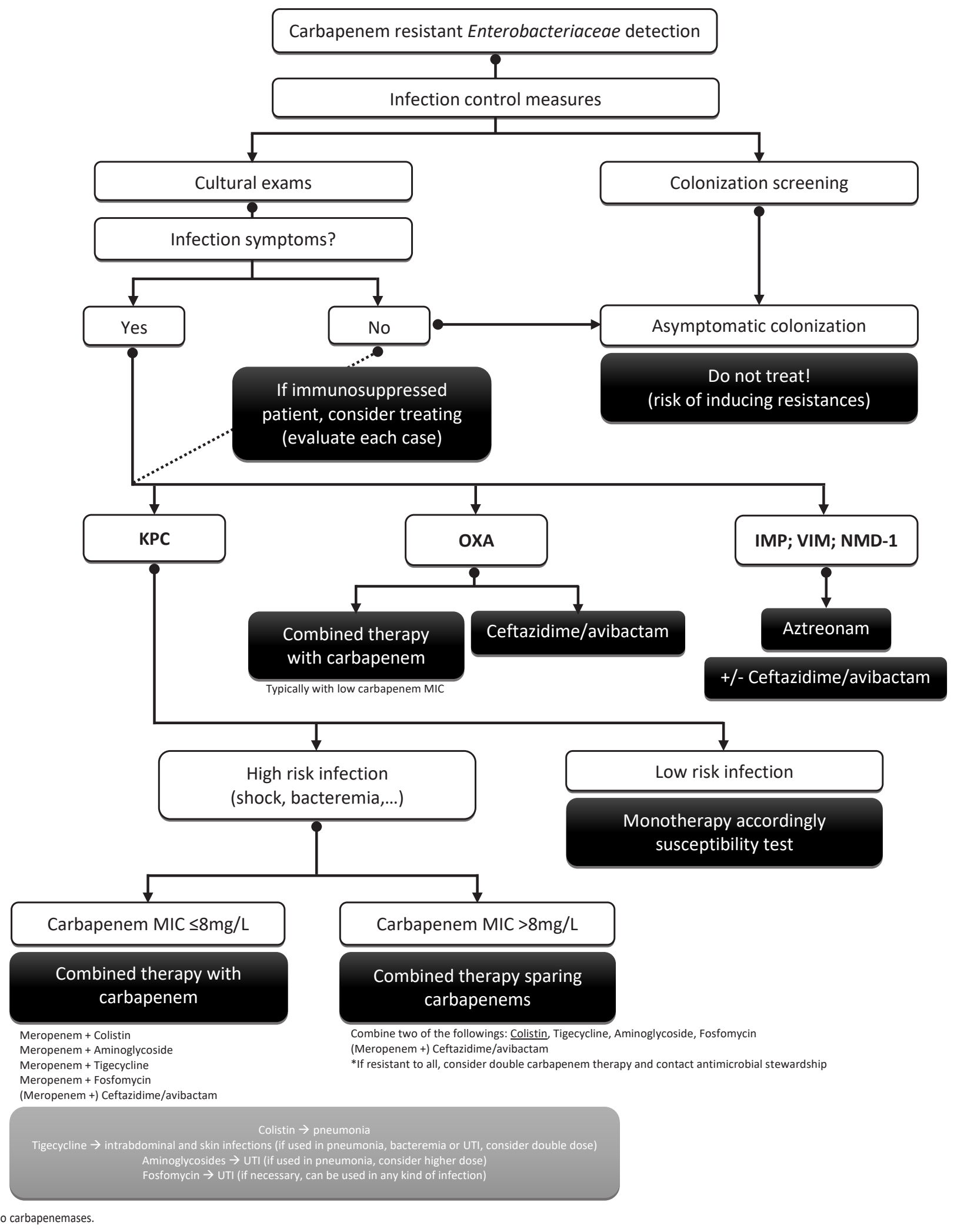


Severe neurological reactions have been reported with ceftazidime, including asterixis, coma, encephalopathy, myoclonus, neuromuscular excitability, seizures, and nonconvulsive status epilepticus.

\section{Meropenem/Vaborbactam}

This new antibiotic is already available in many countries, and soon will be available in Portugal.

Vaborbactam is a new-lactamase inhibitor which has been shown to restore the activity of meropenem against KPC producers.

A small phase 3 trial showed higher rates of clinical cure with meropenem/vaborbactam comparing with the best available therapy, as well as lower rates of nephrotoxicity ${ }^{40}$.

\section{Aztreonam}

This antibiotic is particularly important in MBLs treatment, because MBLs confer resistance to all beta-lactam-type antibiotics except aztreonam $^{41}$.

The problem is that MBL-producing isolates often produce other extended-spectrum beta-lactamases that confer resistance to aztreonam. Although MBLs are not inhibited by any of the available beta-lactamase inhibitors, combining ceftazidime/avibactam and aztreonam can have a complementary effect, as the avibactam can inactivate the other beta-lactamases to render the aztreonam active ${ }^{42-44}$.

\section{How to treat?}

\section{Low risk infections}

These infections (for example, acute simple cystitis) can often be successfully treated with a single active agent, such as an aminoglycoside ${ }^{45}$, meropenem, or colistin ${ }^{4}$. Aminoglycosides can be given as a consolidated, extended-interval dose for 7 to 14 days, depending on response to therapy. Carbapenems can be given if MIC is $4 \mathrm{mg} / \mathrm{L}$ or less. Monotherapy fosfomycin is still the subject of research and trials. Dosage is still unestablished, with probably $3 \mathrm{~g}$ in one single dose insufficient, adding resistance acquisition risk $^{38}$.

\section{High risk infections}

For the most serious infections, the choice of therapy depends on the type of carbapenemase present and the susceptibility profile of the isolate.

For infections caused by Klebsiella pneumoniae carbapenemase (KPC)-producing organisms,

because of the antibiotics' availability, costs and policies, the first-line therapy can be different in each hospital. In Portugal, we still don't have meropenem/vaborbactam available and the cost of ceftazidime/avibactam is too high to give it as a first-line therapy. Thereby, if the carbapenem MIC is $8 \mathrm{mg} / \mathrm{L}$ or less, the first choice should be the combined therapy between a carbapenem and another active antibiotic. If the carbapenem MIC is higher than $8 \mathrm{mg} / \mathrm{L}$, the combined therapy should be chosen accordingly with the susceptibility test, giving priority to colistin as long as the isolate is susceptible ${ }^{4}$. As said before, when the choice is ceftazidime/avibactam, one should consider adding a second agent, typically a carbapenem ${ }^{38}$.

The source of infection can influence the second agent choice: for example, for gastrointestinal tract and skin, tigecycline should be considered; aminoglycosides should be avoided in abscesses, and in pneumonia other options (if available) should be tried, but it can be a good choice in urinary tract.

When beta-lactam agents are used for carbapenemase-producing isolates, prolonged infusion dosing can be considered.

For infections caused by isolates producing metallo-beta-lactamases $(\mathrm{MBL})$, the combination of ceftazidime/avibactam plus aztreonam may be a possible option.

\section{INFECTION CONTROL}

Hospitalized patients, infected or colonized with carbapenemase-producing bacteria, should be placed on contact precautions ${ }^{25,46-49}$.

After discharge, contact precautions should be continued (i.e., during future hospitalizations), given the prolonged colonization with such organisms and the limited treatment options. The period that these measures should be maintained is uncertain, and depending on guidelines that the hospital follows, they are maintained for at least six months (some hospitals maintained them indefinitely), or until there are three negative rectal colonization screenings in a row.

Other standard measures, such as hand hygiene, minimizing the use of invasive devices, and antimicrobial stewardship, are important to infection control in general and likely to limit spread of resistant organisms.

Screening high-risk patients to detect rectal colonization has been suggested as an important infection control modality 46,48,50,51. Although the impact of surveillance itself is difficult to assess, it may be useful in the setting of outbreaks due to carbapenem-resistant organisms.

Disclosure of potential conflicts of interest: none declared

\section{References}

1. Antimicrobial resistance: tackling a crisis for the health and wealth of nations. Available at http:// www.jpiamr.eu/wp-content/uploads/2014/12/AMR-Review-Paper-Tackling-a-crisis-for-the-health-and-wealth-of-nations_1-2.pdf. Accessed May 9, 2019

2. Murray Patrick R, Rosenthal Ken S, Pfaller Michael A. Medical Microbiology. 8th Edition. Philadelphia: Elsevier/Saunders, 2016:251-264

3. Jacoby GA. AmpC ß-lactamases. Clin Microbiol Rev 2009;22(1):161-182

4. Rodríguez-Baño J, Gutiérrez-Gutiérrez B, Machuca I, Pascual A. Treatment of infections caused by extended-spectrum-beta-lactamase-, ampC-, and carbapenemase-producing Enterobacteriaceae. Clin Microbiol Rev 2018;31(2):e00079-e00117

5. Ambler RP. The structure of $\beta$-lactamases. Philos Trans R Soc Lond B Biol Sci 1980;289(1036):321$-331$

6. Yigit $\mathrm{H}$, Queenan AM, Anderson GJ, et al. Novel carbapenem-hydrolyzing beta-lactamase, KPC-1, from a carbapenem-resistant strain of Klebsiella pneumoniae. Antimicrob Agents Chemother 2001;45(4):1151-1161

7. Nordmann P, Cuzon G, Naas T. The real threat of Klebsiella pneumoniae carbapenemase-producing bacteria. Lancet Infect Dis 2009;9(4):228-236 
8. Centers for Disease Control and Prevention (CDC). Vital signs: carbapenem-resistant Enterobacteriaceae. MMWR Morb Mortal Wkly Rep 2013;62(9):165-170

9. Proportion of carbapenem-resistant Klebsiella pneumoniae isolates (\%) in Europe in 2017. Available at https://ecdc.europa.eu/en/antimicrobial-resistance/surveillance-and-disease-data/data-ecdc. Accessed November 20, 2018

10. Yong D, Toleman MA, Giske CG, et al. Characterization of a new metallo-beta-lactamase gene, bla(NDM-1), and a novel erythromycin esterase gene carried on a unique genetic structure in Klebsiella pneumoniae sequence type 14 from India. Antimicrob Agents Chemother 2009;53(12):5046-5054

11. Kumarasamy KK, Toleman MA, Walsh TR, et al. Emergence of a new antibiotic resistance mechanism in India, Pakistan, and the UK: a molecular, biological, and epidemiological study. Lancet Infect Dis 2010;10(9):597-602

12. Centers for Disease Control and Prevention (CDC). Detection of Enterobacteriaceae isolates carrying metallo-beta-lactamase - United States, 2010. MMWR Morb Mortal Wkly Rep 2010;59(24):750

13. Sidjabat H, Nimmo GR, Walsh TR, et al. Carbapenem resistance in Klebsiella pneumoniae due to the New Delhi Metallo- $\beta$-lactamase. Clin Infect Dis 2011;52(4):481-484

14. Centers for Disease Control and Prevention (CDC). Carbapenem-resistant Enterobacteriaceae containing New Delhi metallo-beta-lactamase in two patients - Rhode Island, March 2012. MMWR Morb Mortal Wkly Rep 2012;61(24):446-448

15. Gupta N, Limbago BM, Patel JB, Kallen AJ. Carbapenem-resistant Enterobacteriaceae: epidemiology and prevention. Clin Infect Dis 2011;53(1):60-67

16. Thoms-Rodriguez CA, Mazzulli T, Christian N, et al. New Delhi metallo- $\beta$-lactamase in Jamaica. Infect Dev Ctries 2016;10(2):183-187

17. Herbert S, Halvorsen DS, Leong T, et al. Large outbreak of infection and colonization with gram-negative pathogens carrying the metallo-beta-lactamase gene blalMP-4 at a 320-bed tertiary hospital in Australia. Infect Control Hosp Epidemiol 2007;28(1):98-101

18. Nouér SA, Nucci M, de-Oliveira MP, et al. Risk factors for acquisition of multidrug-resistant Pseudomonas aeruginosa producing SPM metallo-beta-lactamase. Antimicrob Agents Chemother 2005;49(9):3663-3667

19. Schwaber MJ, Klarfeld-Lidji S, Navon-Venezia S, et al. Predictors of carbapenem-resistant Klebsiella pneumoniae acquisition among hospitalized adults and effect of acquisition on mortality. Antimicrob Agents Chemother 2008;52(3):1028-1033

20. Marchaim D, Chopra T, Bhargava A, et al. Recent exposure to antimicrobials and carbapenem-resistant Enterobacteriaceae: the role of antimicrobial stewardship. Infect Control Hosp Epidemiol 2012;33(8):817-830

21. Bratu S, Brooks S, Burney S, et al. Detection and spread of Escherichia coli possessing the plasmid-borne carbapenemase KPC-2 in Brooklyn, New York. Clin Infect Dis 2007:44(7):972-975

22. Hossain A, Ferraro MJ, Pino RM, et al. Plasmid-mediated carbapenem-hydrolyzing enzyme KPC-2 in an Enterobacter sp. Antimicrob Agents Chemother 2004;48(11):4438-4440

23. Peleg AY, Franklin C, Bell JM, Spelman DW. Dissemination of the metallo-beta-lactamase gene blaIMP-4 among gram-negative pathogens in a clinical setting in Australia. Clin Infect Dis 2005;41(11):1549-1556

24. Wei ZQ, Du XX, Yu YS, et al. Plasmid-mediated KPC-2 in a Klebsiella pneumoniae isolate from China. Antimicrob Agents Chemother 2007;51(2):763-765

25. Villegas MV, Lolans K, Correa A, et al. First detection of the plasmid-mediated class A carbapenemase KPC-2 in clinical isolates of Klebsiella pneumoniae from South America. Antimicrob Agents Chemother 2006;50(8):2880-2882

26. Deshpande P, Shetty A, Kapadia F, et al. New Delhi metallo 1: have carbapenems met their doom? Clin Infect Dis 2010;51(10):1222

27. Bratu S, Landman D, Haag R, et al. Rapid spread of carbapenem-resistant Klebsiella pneumoniae in New York City: a new threat to our antibiotic armamentarium. Arch Intern Med 2005;165(12):1430-1435

28. Toleman MA, Biedenbach D, Bennett DM, et al. Italian metallo-beta-lactamases: a national problem? Report from the SENTRY Antimicrobial Surveillance Programme. J Antimicrob Chemother 2005;55(1):61-70

29. Borgmann S, Wolz C, Gröbner S, et al. Metallo-beta-lactamase expressing multi-resistant Acinetobacter baumannii transmitted in the operation area. J Hosp Infect 2004;57(4):308-315

30. Bratu S, Tolaney P, Karumudi U, et al. Carbapenemase-producing Klebsiella pneumoniae in BrookIyn, N.Y.: molecular epidemiology and in vitro activity of polymyxin B and other agents. J. Antimicrob. Chemother 2005;56(1):128-132

31. Daikos GL, Tsaousi S, Tzouvelekis LS, et al. Carbapenemase producing Klebsiella pneumoniae bloodstream infections: lowering mortality by antibiotic combination schemes and the role of carbapenems. Antimicrob Agents Chemother 2014;58(4):2322-2328

32. Gutiérrez-Gutiérrez B, Salamanca E, de Cueto M, et al. Effect of appropriate combination therapy on mortality of patients with bloodstream infections due to carbapenemase-producing Enterobacteriaceae (INCREMENT): a retrospective cohort study. Lancet Infect Dis 2017;17(7):726-734
33. Machuca I, Gutiérrez-Gutiérrez B, Gracia-Ahufinger I, et al. Mortality associated with bacteremia due to colistin-resistant Klebsiella pneumoniae with high-level meropenem resistance: importance of combination therapy without colistin and carbapenems. Antimicrob Agents Chemother 2017;61(8):e00406-e00417

34. Tumbarello M, Trecarichi EM, De Rosa FG, et al. Infections caused by KPC-producing Klebsiella pneumoniae: differences in therapy and mortality in a multicentre study. J Antimicrob Chemother 2015;70(7):2133-2143

35. Bulik CC, Nicolau DP. Double-carbapenem therapy for carbapenemase-producing Klebsiella pneumoniae. Antimicrob. Agents Chemother 2011;55(6):3002-3004

36. Kift EV, Maartens G, Bamford C. Systematic review of the evidence for rational dosing of colistin S Afr Med J 2014;104(3):183-186

37. Li J, Nation RL, Turnidge JD, et al. Colistin: the re-emerging antibiotic for multidrug-resistant Gram-negative bacterial infections. Lancet Infect Dis 2006;6(9):589-601

38. Hawkey PM, Warren RE, Livermore DM, et al. Treatment of infections caused by multidrug-resistant Gram-negative bacteria: report of the British Society for Antimicrobial Chemotherapy/Healthcare Infection Society/British Infection Association Joint Working Party. J Antimicrob Chemother 2018;73(3):iii2-iii78

39. Shields RK, Nguyen MH, Press EG, Chen L, Kreiswirth BN, Clancy CJ. Emergence of ceftazidime-avibactam resistance and restoration of carbapenem susceptibility in Klebsiella pneumoniae carbapenemase producing $K$. pneumoniae: a case report and review of literature. Open Forum Infect Dis 2017:4(3): of $\times 101$

40. Kaye K, Vazquez J, Mathers A, et al. Clinical outcomes of serious infections due to carbapenem-resistant Enterobacteriaceae (CRE) in TANGO II, a phase 3, randomized, multi-national, open-labe trial of meropenem-vaborbactam (M-V) versus best available therapy (BAT). IDWeek 2017, poster 1862

41. Bellais S, Mimoz O, Léotard S, et al. Efficacy of beta-lactams for treating experimentally induced pneumonia due to a carbapenem-hydrolyzing metallo-beta-lactamase-producing strain of Pseudomonas aeruginosa. Antimicrob Agents Chemother 2002;46(6):2032-2034

42. Marshall S, Hujer AM, Rojas LJ, et al. Can Ceftazidime-Avibactam and Aztreonam Overcome $\beta$-Lactam Resistance Conferred by Metallo- $\beta$-Lactamases in Enterobacteriaceae? Antimicrob Agents Chemother 2017;61(4):e02243-e02316

43. Jayol A, Nordmann P, Poirel L, Dubois V. Ceftazidime/avibactam alone or in combination with aztreonam against colistin-resistant and carbapenemase-producing Klebsiella pneumoniae. Antimicrob Chemother 2018;73(2):542-544

44. Shaw E, Rombauts A, Tubau F, et al. Clinical outcomes after combination treatment with ceftazidime/avibactam and aztreonam for NDM-1/OXA-48/CTX-M-15-producing Klebsiella pneumoniae infection. J Antimicrob Chemother 2018;73(4):1104-1106

45. Shields RK, Clancy CJ, Press EG, Nguyen MH. Aminoglycosides for Treatment of Bacteremia Due to Carbapenem-Resistant Klebsiella pneumoniae. Antimicrob Agents Chemother 2016;60(5):3187$-3192$

46. Hirakata Y, Izumikawa K, Yamaguchi T, et al. Rapid detection and evaluation of clinical characteristics of emerging multiple-drug-resistant gram-negative rods carrying the metallo-beta-lactamase gene blalMP. Antimicrob Agents Chemother 1998;42(8):2006-2011

47. Crespo MP, Woodford N, Sinclair A, et al. Outbreak of carbapenem-resistant Pseudomonas aeruginosa producing VIM-8, a novel metallo-beta-lactamase, in a tertiary care center in Cali, Colombia. J Clin Microbiol 2004;42(11):5094-5101

48. Aubron C, Poirel L, Fortineau N, et al. Nosocomial spread of Pseudomonas aeruginosa isolates expressing the metallo-beta-lactamase VIM-2 in a hematology unit of a French hospital. Microb Drug Resist 2005;11(3):254-259

49. Centers for Disease Control and Prevention (CDC). Guidance for control of infections with carbapenem-resistant or carbapenemase-producing Enterobacteriaceae in acute care facilities. MMWR Morb Mortal Wkly Rep 2009;58(10):256-260

50. Walsh TR, Toleman MA, Poirel L, Nordmann P. Metallo-beta-lactamases: the quiet before the storm? Clin Microbiol Rev 2005;18(2):306-325

51. Gijón D, Curiao T, Baquero F, et al. Fecal carriage of carbapenemase-producing Enterobacteriaceae: a hidden reservoir in hospitalized and nonhospitalized patients. J Clin Microbiol 2012;50(5):1558-1563

\section{Correspondence to:}

Marisa Araújo

Rua Abílio Mendes 12, 1500-458 Lisboa

E-mail: dra.marisa.araujo@hotmail.com 